\title{
Corrigendum: Resistin-Induced Endoplasmic Reticulum Stress Contributes to the Impairment of Insulin Signaling in Endothelium
}

\section{OPEN ACCESS}

Approved by:

Frontiers in Pharmacology Editorial

Office,

Frontiers Media SA, Switzerland

*Correspondence:

Ming-Sheng Zhou

zhoums1963@163.com

Specialty section:

This article was submitted to

Cardiovascular and Smooth Muscle

Pharmacology,

a section of the journal

Frontiers in Pharmacology

Received: 18 November 2018

Accepted: 22 November 2018

Published: 10 December 2018

Citation:

Luo J, Huang L, Wang A, Liu Y, Cai R,

Li W and Zhou M-S (2018)

Corrigendum: Resistin-Induced

Endoplasmic Reticulum Stress

Contributes to the Impairment of

Insulin Signaling in Endothelium.

Front. Pharmacol. 9:1446.

doi: 10.3389/fphar.2018.01446

\section{Jun Luo ${ }^{1}$, Lei Huang ${ }^{2}$, Aimei Wang ${ }^{3}$, Yueyang Liu $^{2}$, Ruiping Cai ${ }^{2}$, Weihong $\mathrm{Li}^{3}$ and Ming-Sheng Zhou ${ }^{2 *}$}

${ }^{1}$ Department of Cardiology, The Affiliated Ganzhou Hospital of Nanchang University, Ganzhou, China, ${ }^{2}$ Department of Physiology, Shenyang Medical University, Shenyang, China, ${ }^{3}$ Department of Physiology, Jinzhou Medical University, Jinzhou, China

Keywords: cardiovascular diseases, endothelial nitric oxide synthesis, endoplasmic reticulum stress, resistin, vascular insulin signaling

\section{A Corrigendum on}

Resistin-Induced Endoplasmic Reticulum Stress Contributes to the Impairment of Insulin Signaling in Endothelium

Luo, J., Huang, L., Wang, A., Liu, Y., Cai, R., Li, W., and Zhou, M. S. (2018). Front. Pharmacol. 9:1226. doi: 10.3389/fphar.2018.01226

An author name was incorrectly spelled as Jun Lou. The correct spelling is Jun Luo. The authors apologize for this error and state that this does not change the scientific conclusions of the article in any way. The original article has been updated.

In the published article, there was an error in affiliation 1. Instead of, the Department of Cardiology, Affiliated Ganzhou City Hospital, Nanchang University, it should be, the Department of Cardiology, The Affiliated Ganzhou Hospital of Nanchang University.

In addition, there was an error in the affiliation(s) for Ming-Sheng Zhou. Instead of, 2 Department of Physiology, Shenyang Medical University, Shenyang, China, 3 Department of Physiology, Jinzhou Medical University, Jinzhou, China, they should only have, 2 Department of Physiology, Shenyang Medical University, Shenyang, China. The authors apologize for this error and state that this does not change the scientific conclusions of the article in any way. The original article has been updated.

Conflict of Interest Statement: The authors declare that the research was conducted in the absence of any commercial or financial relationships that could be construed as a potential conflict of interest.

Copyright (C) 2018 Luo, Huang, Wang, Liu, Cai, Li and Zhou. This is an open-access article distributed under the terms of the Creative Commons Attribution License (CC BY). The use, distribution or reproduction in other forums is permitted, provided the original author(s) and the copyright owner(s) are credited and that the original publication in this journal is cited, in accordance with accepted academic practice. No use, distribution or reproduction is permitted which does not comply with these terms. 